\title{
Bubbles emerging from a submerged granular bed
}

\author{
J. A. MEIER $R^{1}$, J. S. JEWELL ${ }^{1}$, C. E. BREN NEN ${ }^{1} \dagger$ \\ AND J. IMBER GER ${ }^{2}$ \\ ${ }^{1}$ Department of Mechanical Engineering, California Institute of Technology, Pasadena, CA 91125, USA \\ ${ }^{2}$ Centre for Water Resources, University of Western Australia, Perth, Australia
}

(Received 3 December 2009; revised 16 August 2010; accepted 16 August 2010)

This paper explores the phenomena associated with the emergence of gas bubbles from a submerged granular bed. While there are many natural and industrial applications, we focus on the particular circumstances and consequences associated with the emergence of methane bubbles from the beds of lakes and reservoirs since there are significant implications for the dynamics of lakes and reservoirs and for global warming. This paper describes an experimental study of the processes of bubble emergence from a granular bed. Two distinct emergence modes are identified, mode 1 being simply the percolation of small bubbles through the interstices of the bed, while mode 2 involves the cumulative growth of a larger bubble until its buoyancy overcomes the surface tension effects. We demonstrate the conditions dividing the two modes (primarily the grain size) and show that this accords with simple analytical evaluations. These observations are consistent with previous studies of the dynamics of bubbles within porous beds. The two emergence modes also induce quite different particle fluidization levels. The latter are measured and correlated with a diffusion model similar to that originally employed in river sedimentation models by Vanoni and others. Both the particle diffusivity and the particle flux at the surface of the granular bed are measured and compared with a simple analytical model. These mixing processes can be consider applicable not only to the grains themselves, but also to the nutrients and/or contaminants within the bed. In this respect they are shown to be much more powerful than other mixing processes (such as the turbulence in the benthic boundary layer) and could, therefore, play a dominant role in the dynamics of lakes and reservoirs.

Key words: bubble dynamics, geophysical and geological flows, granular media

\section{Introduction}

This paper addresses the phenomena associated with the process of gas bubbles emerging from submerged and packed granular beds. The gas may be injected into the bed or formed by the decay of organic material within it. The phenomena are consequential in a number of contexts, including the emission of methane from oceanic seeps (see e.g. Hornafius, Quigley \& Luyendyk 1999; Mau et al. 2007), but here we focus on the process by which methane bubbles are released from lake beds as a result of the decomposition of organic matter in the lake bed (see e.g. 
(a)

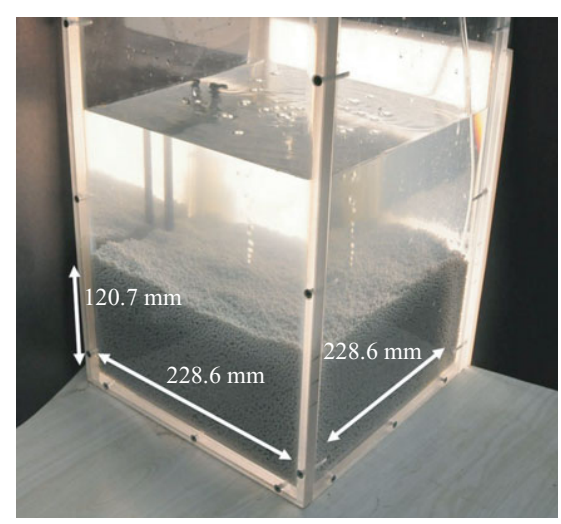

(b)

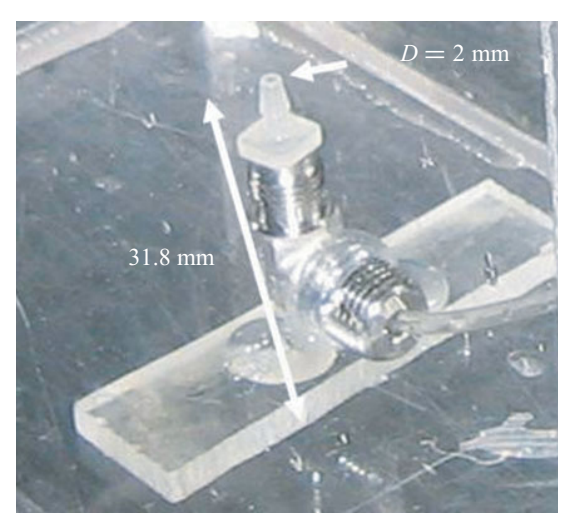

FIGURE 1. (Colour online) Experimental apparatus consisting of the nozzle for air injection $(b)$ embedded in a bed of granular material in a transparent container $(a)$.

Hanson \& Hanson 1996; Schoell, Tietze \& Schoberth 1988; Ostrovsky et al. 2008). Methane gas is 23 times more detrimental per molecule than $\mathrm{CO}_{2}$ in affecting climate change. Therefore, determining the flux of methane from the lake basin could assist in quantifying the effect certain lakes have on the carbon budget (Dean \& Gorham 1998; Strauss 2009). We note that methane levels have increased $100 \%$ within the atmosphere in the past two centuries (Houghton 2007).

Methane bubbles travel up the water column; usually they first shrink because of solution of the methane; later they expand due to the reduction in the pressure particularly as they reach near the surface (McGinnis et al. 2006). Thus, for example, the quantity of methane that is released into the atmosphere in the initial filling phase of reservoirs is dependent on the initial size of the bubble that is released from the lake-bottom sediment. Moreover the mixing caused by bubbles as they rise (Romero, Patterson \& Melack 1996) and the ventilation of deep, nutrient rich water into the surface layer due to the drag of the bubbles is important in lakes that have anoxic hypolimnia (Ostrovsky et al. 2008).

The specific focus of the present paper is on the way bubbles emerge from a submerged granular bed and, secondly, on the mixing which that emergence causes. We should note that there exists a literature on how bubbles rise through granular beds (e.g. Roosevelt \& Corapcioglu 1998; Brooks, Wise \& Annable 1999; Gostiaux, Gayvallet \& Géminard 2002; Pokusaev, Kazenin \& Karlov 2004) though the focus of those studies is on what happens within the bed rather than during emergence. We make reference below to that literature. Here we first describe some simple laboratory experiments that were carried out to examine the phenomena associated with bubble emergence from a granular bed.

\section{Experimental equipment}

The laboratory equipment shown in figure 1 consisted of an acrylic box containing a rectangular granular bed of glass beads $(228.6 \mathrm{~mm}$ wide, $228.6 \mathrm{~mm}$ broad and $120.7 \mathrm{~mm}$ deep). Submerged in this bed was an air injection nozzle (see figure 1) placed in the centre of the bed planform and $31.8 \mathrm{~mm}$ above the floor of the box ( $88.9 \mathrm{~mm}$ below the surface of the bed). The nozzle had a diameter of $2 \mathrm{~mm}$. A nozzle with diameter $1 \mathrm{~mm}$ was also tested, but produced essentially the same results as the 
(a)

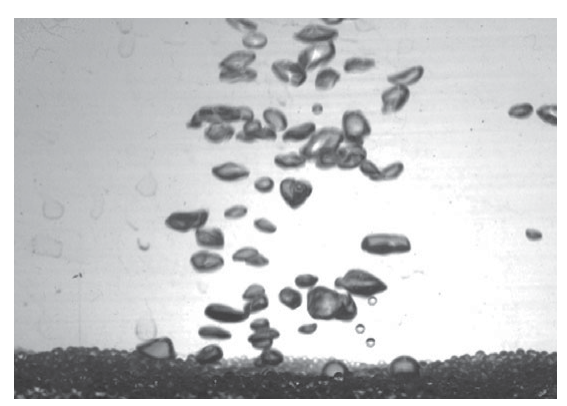

Mode 1 (b)

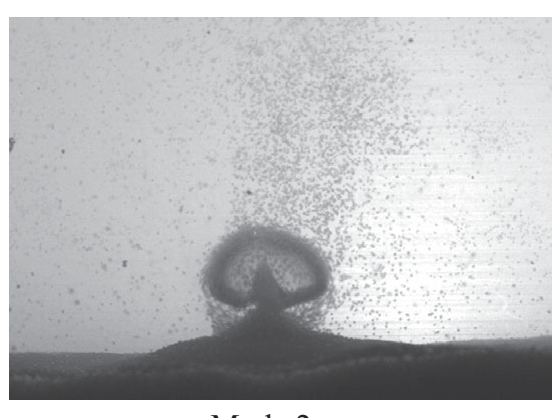

Mode 2

FIGURE 2. Examples of the two modes of bubble release for an air flow rate of $1.01 \mathrm{~min}^{-1}$.

$2 \mathrm{~mm}$ nozzle. These nozzles were supplied with air through a regulator and a Key Instruments FR 2000 Series air flow meter.

Granular beds consisting of six different sizes of spherical glass beads (diameters $0.5,1.25,2,3,4$ and $6 \mathrm{~mm}$ and density $2500 \mathrm{~kg} \mathrm{~m}^{-3}$ ) were used in the experiments. The random loose packing of these beads was about 0.58 . To investigate the effect of particle density on the phenomena, $3 \mathrm{~mm}$ hollow glass beads with a mean density of $1400 \mathrm{~kg} \mathrm{~m}^{-3}$ were also tested.

Photographic observations were made using backlighting with a high-power flood lamp and sheets of light diffusing plastic. Both still photographs and video recordings were made of the bubbles emerging from the bed. A Nanosense MkIII high-speed camera was used to capture video images at 150 frames s$^{-1}$.

\section{Experimental observations}

As the size of the glass beads and the air flow rate were varied, two distinct modes of bubble emergence from the granular bed were encountered. These are exemplified in figure 2. In mode 1, small bubbles percolate up through the interstitial spaces in the granular bed presumably in the manner studied experimentally by Roosevelt \& Corapcioglu (1998), Brooks et al. (1999), Pokusaev et al. (2004) and others. In the present case, these bubbles could emerge from a single site on the bed surface (at low flow rates or with large beads) or multiple sites (at high flow rates or with smaller beads). The latter case is reminiscent of an upside down Pachinko machine. Because of the random lateral migration within the bed, the spread in the bubble emergence sites increased as the bead diameter decreased.

In mode 2, large bubbles emerge from the granular bed, each emergence involving a single large bubble roughly centred over the injection nozzle (though there was some random lateral displacement). As a bubble emerges from the bed, it changes shape and size as it adjusts to a new geometric configuration.

For a given air flow rate, mode 2 would occur for small grain diameters below some critical value, while mode 1 occurred for larger grain sizes. This is illustrated in the photographs of figure 3 for an air flow rate of $11 \mathrm{~min}^{-1}$. At this flow rate beds with grains of 0.5 and $1.25 \mathrm{~mm}$ produced mode 2 emergence, while the larger grains yielded mode 1 behaviour. With the $1.25 \mathrm{~mm}$ glass beads, the transition from mode 2 to mode 1 could also be observed to occur as the air flow rate was increased; this is illustrated in figure 4 , where the mode 1 behaviour at an air flow rate of $0.51 \mathrm{~min}^{-1}$ was observed to change to mode 2 when the flow rate was increased to $1.01 \mathrm{~min}^{-1}$. 
(a)

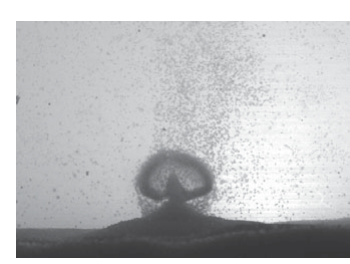

$0.5 \mathrm{~mm}$

(d)

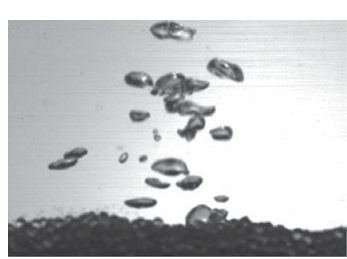

$3.0 \mathrm{~mm}$ (b)

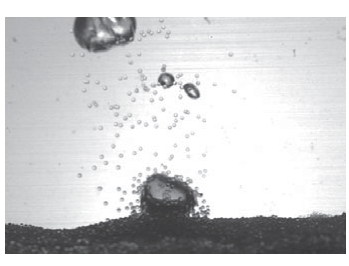

$1.25 \mathrm{~mm}$

(e)

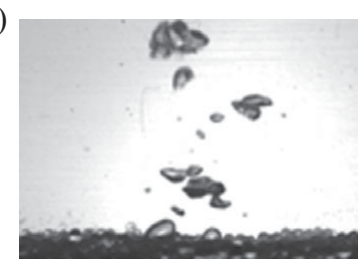

$4.0 \mathrm{~mm}$ (c)

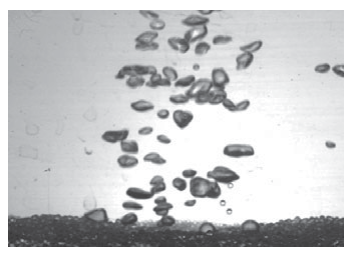

$2.0 \mathrm{~mm}$

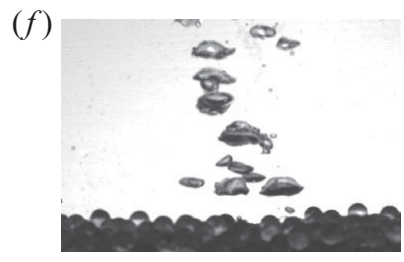

$6.0 \mathrm{~mm}$

FIGURE 3. Photographs of the bubbles emerging from beds of all six sizes of the $2500 \mathrm{~kg} \mathrm{~m}^{-3}$ glass beads (bead diameter shown) for the same air flow rate of $1.01 \mathrm{~min}^{-1}$.

(a)

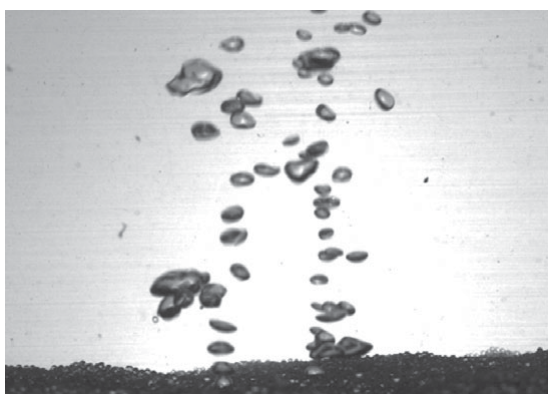

(b)

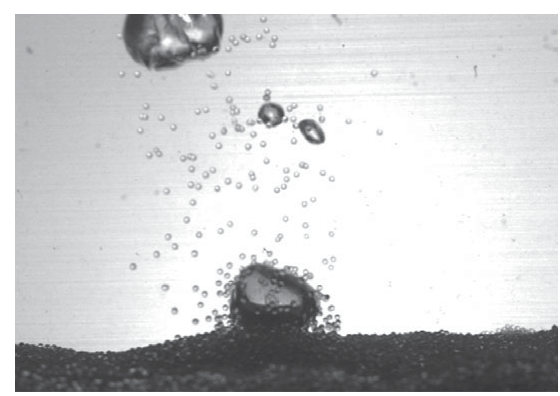

FIGURE 4. Bubbles emerging from a bed of $1.25 \mathrm{~mm}$ glass beads at $0.51 \mathrm{~min}^{-1}(a$, mode 1$)$ and $1.01 \mathrm{~min}^{-1}(b$, mode 2$)$.

We also note that with 0.5 and $1.25 \mathrm{~mm}$ grains there was also a slight trend towards larger bubbles at higher air flow rates as shown in figure 6, but the larger flow rates also tended to cause more grain motion which complicated the picture.

As mentioned earlier, the present tests included an examination of the effect of the particle density by using both solid and hollow $3 \mathrm{~mm}$ glass beads. As shown in figure 5 the solid beads $\left(2500 \mathrm{~kg} \mathrm{~m}^{-3}\right)$ displayed mode 1 behaviour, while the hollow glass beads $\left(1400 \mathrm{~kg} \mathrm{~m}^{-3}\right)$ exhibited mode 2 behaviour. These two photographs also demonstrate that particle fluidization was very different in these two modes; none of the solid beads were fluidized, while many of the hollow beads were elevated high above the surface of the granular bed.

Experiments were also conducted to investigate the effect of the height of the granular bed surface above the air injection point. As this was varied from 95 to $146 \mathrm{~mm}$, there was little change in the mode occurrence or the emerging bubble size.

The average volumetric diameter of the bubbles was evaluated from the measurements of the air flow rate and the number of bubbles produced per second. These bubble diameters are plotted in figure 6 as a function of the grain diameter for each of the air flow rates tested. The occurrence of the two modes of bubble emergence is clearly evident in this graph in which the transition between the two 
(a)

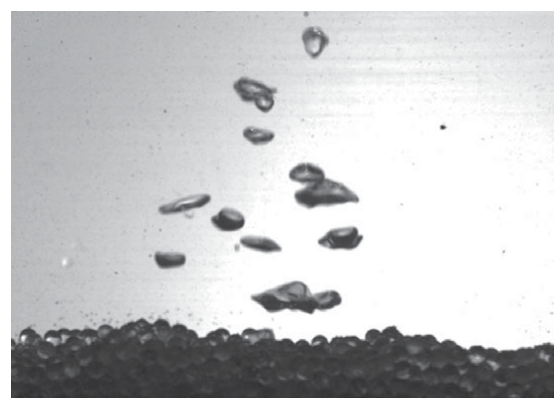

(b)

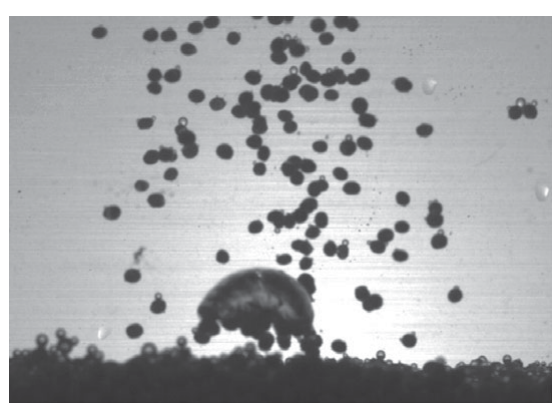

FIGURE 5. Bubbles emerging at $0.61 \mathrm{~min}^{-1}$ from beds of $3 \mathrm{~mm}$ particles. $(a)$ Glass beads with a density of $2500 \mathrm{~kg} \mathrm{~m}^{-3}$. (b) Hollow glass beads with a density of $1400 \mathrm{~kg} \mathrm{~m}^{-3}$.

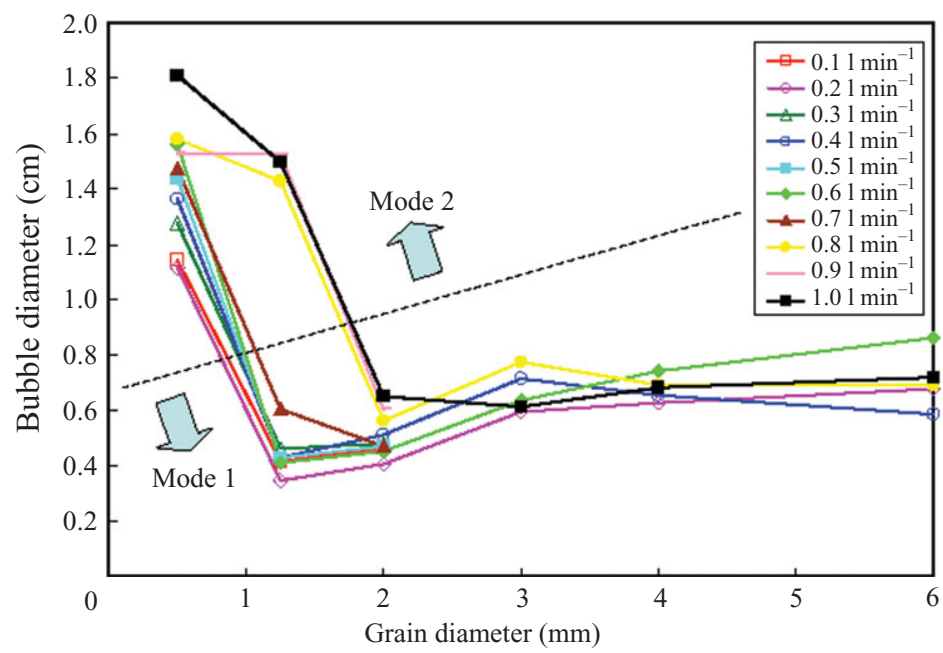

FiguRE 6. (Colour online) Volumetric diameter of the bubbles emerging from the granular beds with $2500 \mathrm{~kg} \mathrm{~m}^{-3}$ glass beads as a function of the bead diameter for 10 different air flow rates (in $1 \mathrm{~min}^{-1}$ ).

modes produces a distinct shift in the size of the emerging bubbles. Figure 6 also illustrates that, within each of the two modes, there was some small increase in the emerging bubble size as the grain size was increased. Also, the emerging bubble size showed some increase with the air flow rate, an effect that could either be due to agglomeration or due to the increase in pressure gradient through the bed due to increased flow rate. Finally, we note that for the large grain sizes, as might be expected the bubble size comes close to the grain size and that the two are essentially equal for the $6 \mathrm{~mm}$ grains.

The effect of particle density is shown in figure 7 for the $3 \mathrm{~mm}$ particles. The solid glass beads $\left(2500 \mathrm{~kg} \mathrm{~m}^{-3}\right)$ exhibited mode 1 behaviour for the full range of air flow rates. On the other hand the hollow glass beads $\left(1400 \mathrm{~kg} \mathrm{~m}^{-3}\right)$ showed mode 2 behaviour for all, but the smallest two flow rates of 0.1 and $0.21 \mathrm{~min}^{-1}$ with the latter being marginal. Note that the distinction between the modes 1 and 2 bubble sizes is consistent throughout figures 6 and 7. In addition, as we discuss below the fluidization was quite different for the two densities of particles. 


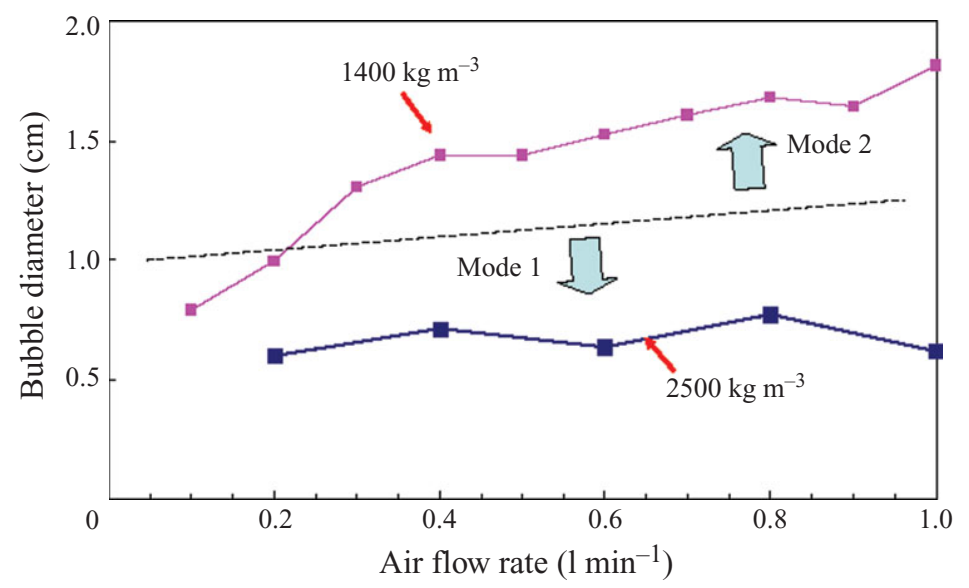

FiguRE 7. (Colour online) The effect of particle density: the volumetric diameter of the emerging bubbles as a function of the air flow rate (in $1 \mathrm{~min}^{-1}$ ) for both the solid $\left(2500 \mathrm{~kg} \mathrm{~m}^{-3}\right)$ and hollow $\left(1400 \mathrm{~kg} \mathrm{~m}^{-3}\right) 3 \mathrm{~mm}$ glass beads.

The above observations of the two modes of emergence and the associated bubble sizes are clearly related to and consistent with the previous observations of the dynamics of bubbles rising in flooded porous beds of granular material. Brooks et al. (1999) review the latter topic, describing the transition observed by Ji et al. (1993) and others from beds of large grains (typically $2 \mathrm{~mm}$ or larger) in which individual bubbles percolate upwards to beds of smaller grains in which accumulations of bubbles move up through channels in the porous medium. Other studies use just one grain size, but provide more detail on the percolation mode; for example, Roosevelt \& Corpcioglu (1998) provide detailed observations of bubbles percolating up through beds of $4 \mathrm{~mm}$ glass beads and Pokusaev et al. (2004) provide both observations and analyses for 3-6 $\mathrm{mm}$ glass bead beds.

There is also some very recent data from natural gas seeps that can be compared with the results of figure 6. Leifer \& Culling (2010) not only made observations of the bubble sizes emerging from the sea floor seeps just off the California coast at Santa Barbara, but also conducted laboratory experiments somewhat similar to those described here using beds with grains ranging from 0.5 to $15.5 \mathrm{~mm}$ in diameter. The laboratory experiments yielded bubbles ranging up to $14 \mathrm{~mm}$ in diameter, a size that is consistent with the results shown in figure 6; however, the change in the mode of emergence was not noted. Leifer and Culling also present data on the bubble sizes from natural seeps most of which emerge from a sandy sea floor; that data indicates bubbles ranging in size from $1.5 \mathrm{~mm}$ to about $12 \mathrm{~mm}$ which would also be consistent with figure 6. Other published data includes the measurements of Ostrovsky et al. (2008) in Lake Kinneret in Israel. Using a sonar technique they found that $90 \%$ of the bubbles ranged from 2.6 to $9 \mathrm{~mm}$ and $50 \%$ ranged from 4 to $6.4 \mathrm{~mm}$ in diameter. These sizes are consistent with both the present observations and those of Leifer and Culling.

\section{Theoretical analyses of the bubble size}

As in previous studies of rising bubbles in flooded porous beds (see e.g. the review of Brooks et al. 1999), the experiments indicate that for granular beds composed 
of large grains, small bubbles simply percolate up through the interstitial liquid. However, when the grains are small the bubbles remain trapped in the interstices and accumulate gas until the agglomerated bubble is large enough for its buoyancy to overcome the surface tension forces holding it in place. Thus, the size of bubble that emerges from the bed is that for whose volume is large enough for the buoyancy force to match the surface tension force. Consequently, the emerging bubble size is determined by the balance of surface tension and buoyancy forces as previously described in the porous media literature (see e.g. Pokusaev et al. 2004). This can be demonstrated in two simple but equivalent ways by considering a large bubble of diameter, $D$, in a packed bed of grains with diameter, $d$, filled otherwise with liquid of density, $\rho$, and surface tension, $S$.

First, the number of grains that intersect the surface of the bubble (assuming $d \ll D$ ) is estimated from the ratio of the surface area of the bubble to the crosssectional area of a grain, or $D^{2} / d^{2}$ (we omit geometric factors of order 1 ). Then an estimate of the total contact line length around all of the bubble-surface grains would be $\pi d\left(D^{2} / d^{2}\right)=\pi D^{2} / d$ and the characteristic surface tension force holding the bubble in place is $\pi S D^{2} / d$. This force is countered by the upward buoyancy force on the bubble. For a packed bed that buoyancy force is simply given by $\pi D^{3} \rho g / 6$, where $g$ is the acceleration due to gravity (note that it is the total volume of the bubble that counts, not just the volume of gas in the bubble for the grains inside the bubble are supported by their own packed structure). If this buoyancy force is then equated to the above estimate of the surface tension force, it leads to a critical bubble diameter, $D_{C}$, of

$$
D_{C}=6 S / \rho g d \text {. }
$$

It is understood that there may be an additional factor of order unity on the right. We note that deviations from this analysis (and the parallel one to follow) are necessary when the granular bed becomes fluidized (as was observed to occur in some regions in some of the experiments described below) for then a mixture density rather than the fluid density should be used and the vertical pressure gradient and buoyancy force would need to be correspondingly modified. This complication is not addressed in the present paper.

Before examining the critical diameter, $D_{C}$, it is worth mentioning a second, but essentially equivalent argument. If the pressure in the gas bubble (assumed uniform) is denoted by $P$, then it can be argued that this will also be the pressure in the water around the waist or mid-section of the bubble (whether this is precisely the case does not effect the argument that follows). Then the pressure in the liquid at the top of the bubble will be $P-\rho g D / 2$ and the pressure in the liquid at the bottom of the bubble will be $P+\rho g D / 2$. Thus, the pressure difference across the liquid-gas interface at the top of the bubble will be $\rho g D / 2$ (lower in the liquid) and the pressure difference across the liquid-gas interface at the bottom of the bubble will be $\rho g D / 2$ (higher in the liquid). These pressure differences will be sustained by the curvature of the gas-liquid interfaces in the interstices. Consequently the radius of curvature of these interstitial gas-liquid interfaces must be $4 S / \rho g D$, where, viewed from the liquid, they are convex at the top of the bubble and concave at the bottom. These pressure differences and interface curvatures cannot be sustained unless the typical intergrain distance is less than twice these radii of curvature. Since the typical intergrain spacing is $d$, it follows that

$$
d<8 S / \rho g D
$$


and, therefore, the critical bubble diameter must be

$$
D_{C}=8 S / \rho g d \text {. }
$$

The two arguments leading to (4.1) and (4.3) are essentially equivalent, and it is, therefore, not surprising that they yield essentially the same result. Clearly we have omitted a factor of order unity that will depend on the geometry of the interstitial menisci and will, therefore, be a complicated function not only of the particle packing and geometry, but also of the contact angle of these menisci on the surfaces of the particles. For example, it seems reasonable to conjecture that hydrophilic particles would have a superior ability to support the required menisci than hydrophobic particles, and therefore, $D_{C}$ would be greater for hydrophilic particles.

The prediction represented by (4.1) and (4.3) is that for a given liquid with a given surface tension ( $S$ and $\rho$ given) the gas bubbles emerging from a granular bed consisting of particles will either be smaller than the typical intergrain distance or will be of a critical size given a relation like (4.1) or (4.3). Moreover, somewhat counterintuitively, that critical size, $D_{C}$, will be larger the smaller the grain size, $d$.

Assuming values for dirty water of $S=0.05 \mathrm{~kg} \mathrm{~s}^{-2}, \rho=1000 \mathrm{~kg} \mathrm{~m}^{-3}, g=9.8 \mathrm{~m} \mathrm{~s}^{-2}$, it follows from (4.1) that the critical bubble size for beds of grains with $d=1.25$ and $2 \mathrm{~mm}$ would be, respectively, $D_{C}=2.4$ and $1.5 \mathrm{~cm}$. These predicted critical bubble sizes are in good agreement with the experimental observations of figure 6 , where the critical or mode 2 bubble size for the $1.25 \mathrm{~mm}$ granular bed is seen to be $1.5 \mathrm{~cm}$ (thus the factor in (4.1) should be 4 rather than 6). In contrast the granular beds with larger particles produce smaller bubbles of the order of $0.7 \mathrm{~cm}$ in size (note the validity of the $d \ll D$ assumption begins to fail for the larger grains). Also note from figure 7 that, in accord with the absence of the particle density in the expressions (4.1) and (4.3), the particle density had little effect on the emerging bubble sizes in the two modes.

In summary, the theoretical prediction that appears to conform with the experimental observations is that bubbles emerging from a bed of grains smaller than some transitional size, will have a diameter, $D_{C}=C S / \rho g d$ (where the constant $C$ has a value of about 4 according to the experiments). Beds with larger grains will, on the other hand produce bubbles with a size smaller than or roughly equal to the intergrain spacing. It follows from these two observations that the transitional grain size, $d_{T}$, occurs when $D_{C} \approx d_{T}$, and therefore, $d_{T}=(C S / \rho g)^{1 / 2}$. With $C=4, S=0.05 \mathrm{~kg} \mathrm{~s}^{-2}$, $\rho=1000 \mathrm{~kg} \mathrm{~m}^{-3}, g=9.8 \mathrm{~m} \mathrm{~s}^{-2}$, this yields $d_{T}=1.4 \mathrm{~mm}$ in close agreement with the observed transitional grain size. As noted earlier they are also consistent with previous studies of bubbles rising within porous media.

\section{Particle movement}

The movement of the bubbles, particularly the large mode 2 bubbles, through and out of the bed clearly caused significant local fluidization of the bed. Particles were carried upwards in the wake of the mode 2 bubbles and caused a substantial increase in the mixing in the neighbourhood of the surface of the granular bed. (We should add parenthetically that, at the start of each of our experiments with the $0.5 \mathrm{~mm}$ glass beds, one could visually distinguish a circular region on the surface of the granular bed that appeared first around the central emergence site and then grew with time both horizontally and vertically into a conical region that extended from the injection point up to the granular surface. It seemed to represent a boundary between the conical region in which the grains were periodically fluidized and the 
outer, undisturbed regions of the granular bed. The effect was apparent to the naked eye, but was not captured in the photographs due to the relative opacity of the small beads and the backlit nature of the illumination. The effect was not, however, noticeable for $1.25 \mathrm{~mm}$ and larger beads.)

Since the fluidization of individual grains above the mean surface of the granular bed is important in evaluating the degree of near-bed mixing caused by the bubbles, an attempt was made to approximately quantify this characteristic through analysis of the videos. For simplicity and in order to compare the mixing of particles caused by bubble emergence in lakes and reservoirs with the mixing caused by other mechanisms we have chosen to characterize the mixing as a convection/diffusion process in a manner similar to the classic treatment of saltation in river beds (see e.g. Vanoni 1975). Thus we characterize the extent of upward particle mixing by a vertical diffusivity, both for simplicity and in order to compare this with measured particle diffusivities due to other mechanisms. In using this characterization, we model the vertical, time-averaged particle distribution in the liquid above the particle bed as a balance between the downward convection of the particles due to their terminal velocity, $w$, and an upward diffusion of particles caused by the unsteady mixing resulting from bubble emergences. We characterize the latter by a diffusivity, $\kappa$. Further study may be needed to assess the limitations of this convection/diffusion model in the present context.

Given the convection/diffusion model, it follows that the time-averaged number density of the particles will decay exponentially with elevation, $y$, above the undisturbed bed surface like $c_{0} \exp (-w y / \kappa)$, where $c_{0}$ denotes the concentration of fluidized particles just above the surface of the granular bed. Then the typical thickness of the layer containing fluidized particles, $\delta$, is proportional to $\kappa / w$. It follows that if we estimate from the videos, values of the average particle concentration at a number of elevations, $y$, and fit those into an exponential curve to determine $w / \kappa$, then the effective diffusivity, $\kappa$, will follow knowing the terminal velocity, $w$. Following this approach, the videotaped images (taken at 150 frames $^{-1}$ ) were processed by dividing the video frame into horizontal strips typically about $3 \mathrm{~mm}$ in height. The number of particles appearing in each strip were counted for the number of frames sufficient to obtain a time-averaged concentration of particles at that elevation. Then this counting was repeated for between four and six different strips vertically distributed over the video frame. These four to six values were then fitted to the exponential function in $y$ in order to obtain the values of $\kappa / w$. To extract the diffusivity, $\kappa$, a terminal velocity, $w$, was then needed for each of the particle sizes; for this purpose calculated terminal velocities for single particles in an infinite fluid were used. For the solid glass beads these were calculated to be $w=13.5,21.4,27.0,33.0,38.2$ and $47.0 \mathrm{~cm} \mathrm{~s}^{-1}$, respectively, for particle sizes $d=0.5,1.25,2,3,4$ and $6 \mathrm{~mm}$. These velocity calculations assumed a coefficient of drag of 0.5 which is roughly appropriate for all these particle sizes over the range of Reynolds numbers involved (Brennen 1995, 2005). It is self-evident this was an extremely laborious process and one that was subject to significant uncertainty, especially during the counting of the smallest particles (thus more accurate terminal velocities taking into account, e.g. the water velocities induced by the bubble motion would not be consequential).

However, despite this uncertainty it is clear that mode 2 cases (all the $d=0.5 \mathrm{~mm}$ data and the data for $d=1.25 \mathrm{~mm}$ when the air flow rate is greater than $0.71 \mathrm{~min}^{-1}$ ) yield much larger diffusivities than the mode 1 cases. Moreover, all the 3, 4 and $6 \mathrm{~mm}$ particle beds showed zero particle fluidization, and therefore, $\kappa \approx 0$.

In comparing the diffusivities of figure 8 with the diffusion caused by other mechanisms (e.g. by the turbulent benthic boundary layer at the bottom of a lake 


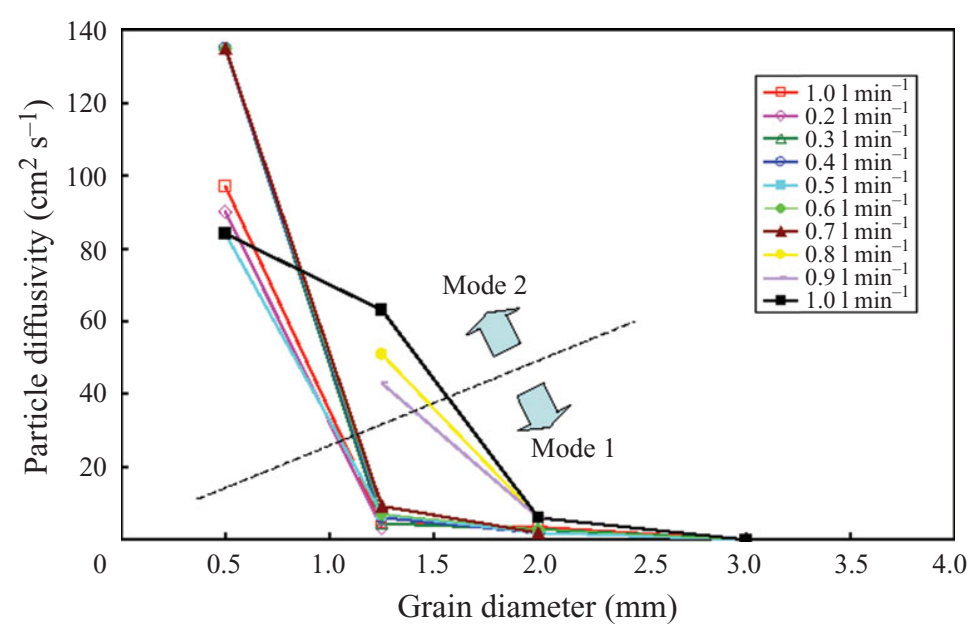

FIGURE 8. (Colour online) Particle diffusivity as a function of the grain diameter $\left(2500 \mathrm{~kg} \mathrm{~m}^{-3}\right.$ glass beads) for all 10 air flow rates tested. Note that there is substantial uncertainty of about $30 \%$ in the estimated diffusivities derived from the experimental observations.

Yeates \& Imberger 2003) we need to bear in mind that these would apply above each bubble emergence site. Therefore, effective bottom-averaged diffusivities would be smaller when the population of bubble emergence sites is factored in and the effect is averaged over the area of the granular bed. However, to put the above diffusivities in perspective we note that typical vertical diffusivities due to turbulence in the benthic boundary layer range up to $1.0 \mathrm{~cm}^{2} \mathrm{~s}^{-1}$ (Yeates \& Imberger 2003), and therefore, just a few bubble emergence sites per square metre could significantly alter the vertical mixing in the benthic boundary layer. (Moreover, these diffusivities are huge when compared with the typical molecular diffusivities for gases in water which are of the order of $2 \times 10^{-5} \mathrm{~cm}^{2} \mathrm{~s}^{-1}$.)

We could anticipate the diffusivity resulting from the two modes as follows. Since the typical mode 2 bubble rise velocity is roughly given by $(g D)^{1 / 2}$ and the typical dimension of the process would be the bubble size, $D$, we could, at least on dimensional grounds, anticipate a diffusivity for the mode 2 bubbles of the order of $g^{1 / 2} D^{3 / 2}$. With a typical mode 2 bubble size of $D=1.5 \mathrm{~cm}$ this yields a diffusivity of $57 \mathrm{~cm}^{2} \mathrm{~s}^{-1}$ which is in very good agreement with the measured mode 2 diffusivities in figure 8 . The diffusivities for mode 1 are smaller because both the size and velocity for these bubbles are smaller.

\section{Magnitude of the fluidized particle flux}

The estimates of the particle diffusivities graphed in figure 8 represent one consequence of the mixing induced by the emerging bubbles. However, the diffusivity does not reflect the magnitude (as opposed to the extent) of the mixing. That magnitude was evaluated from the exponential fits by first estimating the number flux of particles at the granular bed surface, $w c_{0}$, and then multiplying by the single particle volume to obtain a particle volume flux at the granular surface. The particle number flux at (or rather just above the granular bed), $c_{0}$, was evaluated manually from the videos in the one of the following two different ways depending on the particle size. For the larger particles it was possible to count the number of particles descending back to the surface of the granular bed during a substantial interval of 


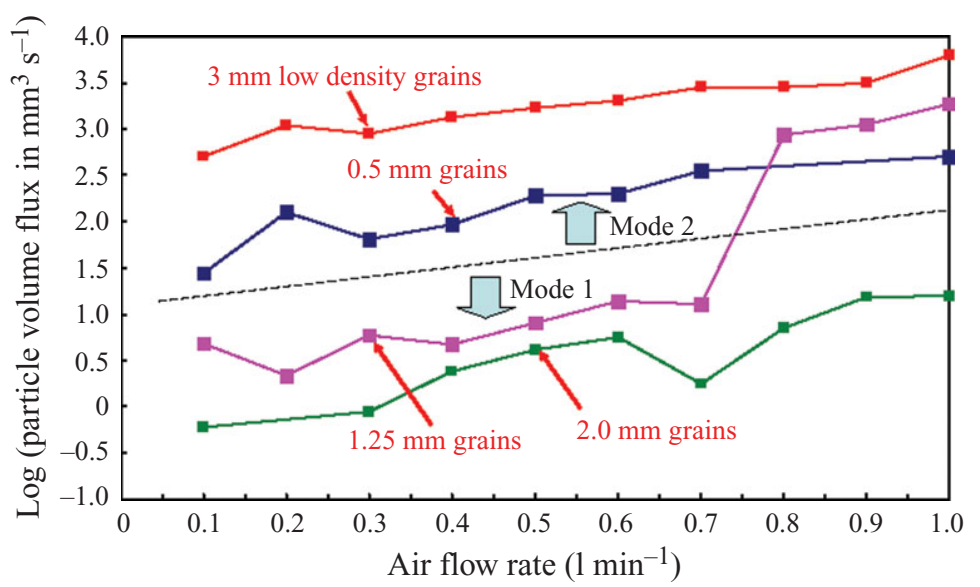

Figure 9. (Colour online) Particle volume flux at the grain bed surface (in $\mathrm{mm}^{3} \mathrm{~s}^{-1}$ ) as a function of the air flow rate (in $1 \mathrm{~min}^{-1}$ ) for the $0.5,1.25$ and $2.0 \mathrm{~mm}$ solid glass beads $\left(2500 \mathrm{~kg} \mathrm{~m}^{-3}\right)$ and for the $3.0 \mathrm{~mm}$ hollow glass beads $\left(1400 \mathrm{~kg} \mathrm{~m}^{-3}\right)$. Note that there is substantial uncertainty of about a factor of two in these particle volume flux derived from the experimental observations.

time (usually about $10 \mathrm{~s}$ ). However, this was impossible for the smaller particles, 0.5 and $1.25 \mathrm{~mm}$ in diameter, and the following alternative procedure was used in those cases. In a time-averaged sense the upward and downward fluxes of particles are equal and are estimated as one half of the local concentration (measured as described in $\S 5$ ) multiplied by the typical particle velocity (for which the individual terminal velocity is assumed to be representative). The resulting values of the particle volume flux just above the granular surface are clearly crude estimates subject to considerable uncertainty estimated to be about a factor of 2 .

The particle volume fluxes for the $0.5,1.25$ and $2.0 \mathrm{~mm}$ solid glass particles are presented in figure 9, where they are plotted against the air flow rate (in the cases of beds with 2, 3, 4 and $6 \mathrm{~mm}$ solid particles the flux was essentially zero). Figure 9 shows that the particle flux is approximately proportional to the air flow rate for a given particle size, particle density and mode. It also indicates that mode 2 emergences produce substantially greater mixing than the mode 1 emergences, in part because of the episodic nature of the mode 2 emergence, but also because the particles are smaller and the bubbles are larger. Also note that the particle flux for the $1.25 \mathrm{~mm}$ particles exhibits a substantial order of magnitude increase as it transitions from modes 1 to 2 .

As could be expected, figure 9 also shows that the particle density (or more accurately the particle buoyancy) has a large effect on the particle fluidization as can be seen by the large particle volume flux that occurs with the $3 \mathrm{~mm}$ hollow glass beads; in contrast essentially no fluidization occurred with the $3 \mathrm{~mm}$ solid glass beads.

To further investigate and non-dimensionalize the particle volume flux, figure 10 presents the ratio of the particle volume flux (figure 9) to the volume flux of air as a function of the dimensionless parameter, $\Gamma=D /\left(\rho_{P} / \rho_{L}-1\right) d$, which is constructed as follows. The typical net force on a particle due to its weight and its buoyancy would be $\pi d^{3} g\left(\rho_{P}-\rho_{L}\right) / 6$. Counteracting this would be the typical upward drag force on a particle due to the fluid motion induced by the bubbles. Using a typical fluid velocity due to the bubbles of $(g D)^{1 / 2}$ and neglecting factors of order 1 (including the drag coefficient) this yields a drag of order $\rho_{L} d^{2} g D$. Taking the ratio of this to the 


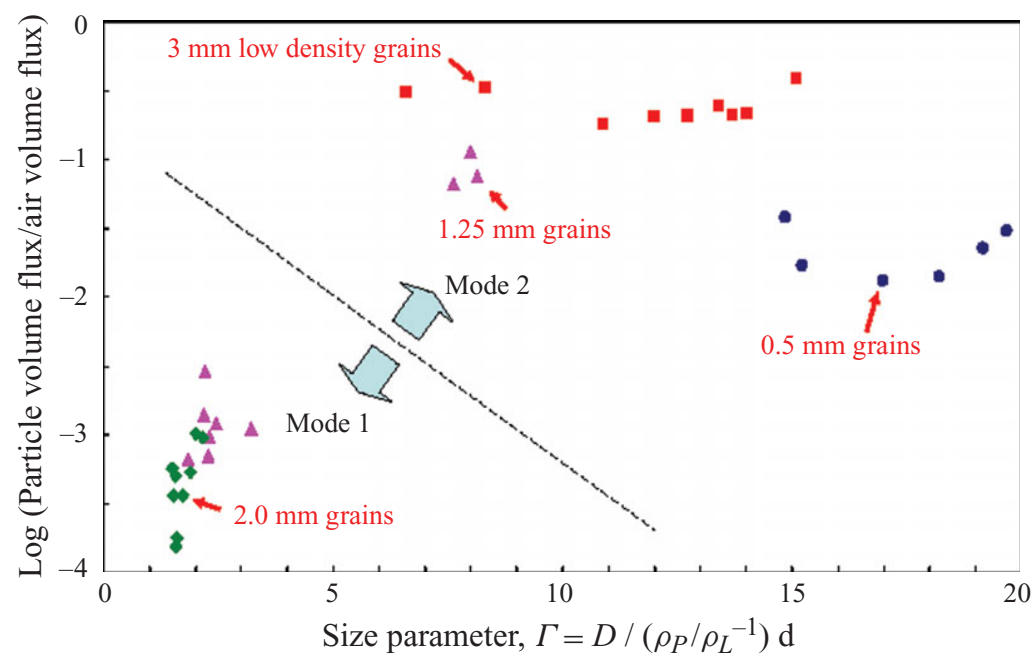

FIGURE 10. (Colour online) Non-dimensional version of figure 9 in which the particle volume flux at the grain bed surface divided by the air flow rate is plotted as a function of the size parameter, $\Gamma$, for the $0.5,1.25$ and $2.0 \mathrm{~mm}$ solid glass beads $\left(2500 \mathrm{~kg} \mathrm{~m}^{-3}\right)$ and for the $3.0 \mathrm{~mm}$ hollow glass beads $\left(1400 \mathrm{~kg} \mathrm{~m}^{-3}\right)$.

net weight/buoyancy produces the parameter $\Gamma$ and we argue that the dimensionless particle flux should be primarily a function of this parameter. Figure 10 shows that this is roughly true. At the higher $\Gamma$ values associated with the mode 2 emergences, the dimensionless particle flux approaches a value of order unity as one would expect. (Perhaps the vertical ordinate could be further modified by the density ratio in order to produce a better correlation.) On the other hand, for the lower mode $1 \Gamma$ values the dimensionless particle flux falls rapidly towards zero as the ratio $D / d$ becomes small.

\section{Nutrient/contaminant diffusivity}

It is well established that bubble plumes in lakes and reservoirs will cause destratification by enhancing vertical mixing. Injected bubble plumes are often used for this purpose and naturally occurring plumes resulting, for example, from methane seeps in lake beds have also been shown to enhance the vertical mixing (see e.g. Romero et al. 2008). However, the present paper focuses on another mechanism generated by bubble seeps namely the mixing generated as a result of the emergence of bubbles from a granular lake bed into the benthic layer of a lake or reservoir. Using these laboratory experiments we seek to demonstrate that the bubble emergence process induces mixing between the interstitial fluid within the granular bed and the liquid above the bed that is large enough to be significant (and perhaps dominant) in lakes and reservoirs with bubble seeps.

We argue that the nutrient/contaminant mixing from the bed into the liquid above the bed (that results from the bubble emergence) will be governed by the same diffusivities as the particles though, of course, the nutrient/contaminant will not settle back down as the particles do. If this is the case then the diffusivities of $\S 8$ imply that bubble emergence is a major contributor to the mixing of nutrient/contaminant from the bed into the liquid overhead. The diffusivity magnitudes of order of $100 \mathrm{~cm}^{2} \mathrm{~s}^{-1}$ 
in the vicinity of the emergence are substantially larger than the typical diffusivities due to turbulence in the benthic boundary layer (order $1 \mathrm{~cm}^{2} \mathrm{~s}^{-1}$ or less) so that even quite a sparse distribution of seepage points could be quite significant. Perhaps this result is not surprising if one views Leifers interesting videos of the surface turbulence produced by the methane seeps in Californias Santa Barbara channel (http://www. bubbleology.com/BubbleologyFrame.html).

It is well known that nutrients contained in algae are re-released into a soluble form when these algae fall to the lake bottom and are broken down by bacteria. This process is called re-mineralization and occurs in the sediments at the lake bottom whenever the oxygen levels drop below a critical value; this is also the process that generates the methane responsible for the bubbles that are the subject of this paper. Depending on the lake, the portion of the nutrients available to primary production in the surface layer, may range from zero to almost one. For the nutrients dissolved in the interstitial water of the sediments to become available to the primary production in the surface layer, first they must be expelled from the bottom sediment into the overlying water and then be transported up into the surface layer. To date investigators have assumed that a thin molecular boundary layer forms at the sediment-water interface, across which nutrients are transported by molecular diffusion; this logic had implied that the molecular flux limited the rate at which nutrients were available to the lake. From the above analysis, it is clear that the expulsion is not a limiting diffusive process, but rather is aided by bubble ventilation; this mechanism carries the pore water to the sediment surface, where it is diffused into the benthic boundary layer by the bubble generated diffusion. From figure 8 we can estimate that the bubbles will mix such nutrients into the first few centimetres of the water column. Now, in a stratified lake, basin-scale internal waves sustain a turbulent benthic boundary layer (Lemckert et al. 2004; Marti \& Imberger 2006) with diffusivities ranging from 0.1 to $10 \mathrm{~cm}^{2} \mathrm{~s}^{-1}$. These values are small compared to those this paper shows are generated by the bubbles themselves in the first few centimetres; the nutrients would thus be mixed into the benthic boundary layer in times of around a minute (see Yeates \& Imberger 2003). Recently, Shimizu \& Imberger (2008) have shown that the energy sustaining the turbulence in the benthic boundary layer is channelled from the wind, to the basinscale waves to the benthic boundary layer in a way that ensures an almost continuous turbulent state in the benthic boundary layer, independent of the seasonal variation of the wind; the benthic boundary layer in the deep part of the lake is thus in a state of readiness to receive nutrients from the sediments, whenever the chemical state of the sediments is such as to liberate nutrients via the bubble stirring. It still remains to explain how nutrients resident in the deep benthic boundary layer reach the surface layer, where they become available to the primary production. Marti \& Imberger (2006) showed that a baroclinic density volume flux is set-up in the benthic boundary layer whenever a nonlinear density stratification exists in the lake, a condition that is nearly always present. Recently, Nakayama \& Imberger (2010) have clarified this volume flux and showed that it may be sustained not only by a nonlinear density gradient, but also by differential mixing in the benthic boundary layer and nonlinear basin-scale internal wave breaking over a shoaling lake bottom. The current work has thus supplied the limiting link in the availability of re-generated nutrients.

\section{Conclusions}

The experiments on the emergence of gas bubbles from a submerged granular bed show that there are two modes of bubble emergence. In beds consisting of larger 
particles the gas bubbles simply percolate up through the granular interstices and emerge with a size comparably to the grain size. However, below a certain critical grain size surface tension forces halt the percolation and bubbles agglomerate in the bed until the buoyancy force on that much larger, agglomerated bubble overcomes the surface tension force. These observations of the two modes of emergence and the associated bubble sizes are clearly related to and consistent with previous observations of the dynamics of bubbles rising in flooded porous beds of granular material. Analyses similar to those in the existing porous media literature demonstrate that the mode 2 bubbles have a predictable size that increases as the grain size decreases. That theoretical prediction of the critical size agrees with the experimental observations.

These mechanisms of bubble emergence are important in the context of the methane or carbon dioxide bubbles that can form in the sediment in lakes and reservoirs or emerge as seeps in oceans or lakes. Indeed the bubble sizes observed in the present experiments are in accord with the size observations from a variety of lakes and ocean waters. However, the distinction between the two modes of emergence does not appear to have been recognized before.

In these natural processes, the emerging bubble size and other features are important for several reasons. First, as demonstrated by McGinnis et al. (2006) the amount of methane that is released to the atmosphere is very much a function of the initial emerging bubble size since small bubbles are often completely dissolved before they reach the water surface and consequently, the larger mode 2 bubbles could be a dominant contributor to the atmospheric release.

However, this paper also demonstrates that the bubble emergence process is likely to be important for a second reason. Depending on the size of the grains, the emerging bubbles can temporarily fluidize a substantial number of grains. From videos of the present laboratory experiments, we quantify both the extent and magnitude of this fluidization. By modelling the fluidization as a balance between particle diffusion by the unsteady bubble motions and resettling at a particle terminal velocity, we evaluate the effective diffusivity produced by the bubble emergence. The diffusivity decreases very rapidly with the grain size. We note that the calculated diffusivities above a bubble emergence site are orders of magnitude larger than the diffusivities that could result from other mixing processes such as that due to turbulence in a benthic boundary layer. Consequently, it does not take a very large population of bubble emergence sites for this to become the dominant mixing process in the lower part of the benthic boundary layer.

The magnitude of the particle fluidization flux is also presented and also shows a radical decline with increasing grain size. Moreover, the mode 2 emergence process shows a substantially larger fluidization flux magnitude than do the gentler mode 1 emergences.

Finally, we also conjecture that the nutrient or contaminant that may be contained in the sediment of a lake, reservoir or ocean will be carried up into the overlying water by this particle mixing process, and therefore, that the details of the emergence process and the particle motions it generates are important in this context. Perhaps, one could use the diffusivities and flux magnitudes calculated here to estimate the rate of mixing of interstitial nutrient/contaminant into the overlying water.

C.E.B. would like to acknowledge the financial support from CWR for a three month visit in 2009 to the Centre for Water Research, University of Western Australia. This paper also forms publication $2282 \mathrm{JM}$ in the CWR publications series. 


\section{REFERENCES}

Brennen, C. E. 1995 Cavitation and Bubble Dynamics. Oxford University Press.

Brennen, C. E. 2005 Fundamentals of Multiphase Flow. Cambridge University Press.

Brooks, M. C., Wise, W. R. \& Annable, M. D. 1999 Fundamental changes in in situ air sparging flow patterns. Ground Water Monit. Remediat. 19 (2), 105-113.

Dean, W. E. \& Gorham, E. 1998 Magnitude and significance of carbon burial in lakes, reservoirs, and peatlands. Geology 26, 535-538.

Gostiaux, L., Gayvallet, H. \& GÉminard, J.-C. 2002 Dynamics of a gas bubble rising through a thin immersed layer of granular material: an experimental study. Granular Matter 4, 39-44.

Hanson, R. S. \& Hanson, T. E. 1996 Methanotrophic bacteria. Microbiol. Rev. 60 (2), 439-471.

Hornafius, J. S., Quigley, D. \& LuYendyK, B. P. 1999 The world's most spectacular marine hydrocarbon seeps (Coal Oil Point, Santa Barbara Channel, California): quantification of emissions. J. Geophys. Res. 104, 20703-20711.

Houghton, R. A. 2007 Balancing the global carbon budget. Annu. Rev. Earth Planet. 35, 313347.

Ji, W., Dahmani, A., Ahlfeld, D. P., Lin, J. D. \& Hill, E. 1993 Laboratory study of air sparging: air flow visualization. Ground Water Monit. Remediat 14 (4), 115-126.

Leifer, I. \& Culling, D. 2010 Formation of seep bubble plumes in the Coal Oil Point Seep field. Geo-Mar. Lett. 30, 339-353.

Lemckert, C., Antenucci, J., Saggio, A. \& Imberger, J. 2004 Physical properties of turbulent benthic boundary layers generated by internal waves. ASCE J. Hydraul. Engng doi:10.1061/(ASCE)0733-9429(2004)130:1(58).

Marti, C. L. \& Imberger, J. 2006 Dynamics of the benthic boundary layer in a strongly forced stratified lake. Hydrobiologia 568, 217-233.

Mau, S., Valentine, D. L., Clark, J. F., Reed, J., Camilli, R. \& Washburn, L. 2007 Dissolved methane distributions and air sea flux in the plume of a massive seep field, Coal Oil Point, California. Geophys. Res. Lett. 34, L22603.

McGinnis, D. F., Greinert, J., Artemov, Y., Beaubien, S. E. \& Wuest, A. 2006 Fate of rising methane bubbles in stratified waters: how much methane reaches the atmosphere? J. Geophys. Res. 111, C09007.

Nakayama, K. \& Imberger, J. 2010 Residual circulation due to internal waves shoaling on a slope. J. Phys. Oceanogr. submitted.

Ostrovsky, I., McGinnis, D. F., Lapidus, L. \& Eckert, W. 2008 Quantifying gas ebullition with echosounder: the role of methane transport by bubbles in a medium-sized lake. Limnol. Oceanogr.: Methods 6, 105-118.

Pokusaev, B. G., Kazenin, D. A. \& Karlov, S. P. 2004 Immersion tomographic study of the motion of bubbles in a flooded granular bed. Teoreticheskie Osnovy Khimicheskoi Tekhnologii 38 (6), 595-603 (translation in Theor. Found. Chem. Engng 38 (6), 561568).

Romero, J. R., Patterson, J. C. \& Melack, J. M. 1996 Simulation of the effect of methane bubble plumes on vertical mixing in Mono Lake. Aquat. Sci. 58 (3), 211-223.

Roosevelt, S. E. \& CoRAPCioglu, M. Y. 1998 Air bubble migration in a granular porous medium: experimental studies. Water Resour. Res. 34 (5), 1131-1142.

Schoell, M., Tietze, K. \& Schoberth, S. M. 1988 Origin of methane in Lake Kivu (East-Central Africa). In: M. Schoell (Guest editor), Origins of methane in the Earth. Chem. Geol. 71, 257-265.

Shimuzu, K. \& Imberger, J. 2008 Energetics and damping of basin-scale internal waves in a strongly stratified lake. Limnol. Oceanogr. 53 (4), 1574-1588.

Strauss, R. 2009 An assessment of sediment diagenesis modelling by the computational aquatic ecosystem dynamics model. Report for Centre for Water Research, University of Western Australia.

VANONI, V. A. 1975 Sedimentation Engineering. ASCE Manuals and Reports on Engineering Practice No. 54. ASCE.

YeAtes, P. S. \& Imberger, J. 2003 Pseudo two dimensional simulations of internal and boundary fluxes in stratified lakes and reservoirs. Intl. J. River Basin Manage. 1 (4), 297-319. 